\title{
Histologische Untersuchung über das Schicksal intravenös infundierten Fettes im Organismus.
}

\author{
Von \\ Shigeru Kimura. \\ (木 林茂) \\ (Aus der medizinischen Klinik von Prof. S. Yamakau a \\ an der Kaiserlichen Tohoku-Universität zu Sendai.)
}

Einleitung.

Es ist schon von manchen Autoren erwiesen, dass Fett sowie Lipoid, wie es bei verschiedenen anorganischen Salzen oder anderen organischen Substanzen der Fall ist, sehr bald nach deren intravenöser Infusion aus der Blutbahn verschwinden. Dies konnten Bondi und Neumann schon ${ }^{1)} 30$ Minuten nach der intravenösen Injektion mit Soda hergestellter Neutralfettemulsion bestätigen. Saxl und Donath ${ }^{2}$ fanden auch beim Menschen, dass fein zerstäubte Öltröpfchen gleich nach intravenöser Injektion nicht mehr in der Blutbahn nachzuweisen waren.

In dieser Klinik hat auch Nomura $a^{3)}$ früher durch chemische Untersuchung festgestellt, dass hoch dispergierte Fettemulsion, die den Kaninchen intravenös injiziert wurde, grösstenteils gleich danach die Zirkulation verliess und nur zum kleinen Bruchteil verhältnismässig lange in der Blutbahn kreiste.

Was für ein Schicksal denn dieses der Zirkulation entgangene Fett weiter teilen soll, ist von verschiedenen Autoren eifrigst erforscht worden. Bodo und Schäffer ${ }^{4)}$ berichteten durch Bestimmung des in den verschiedenen Organen verteilten Jodgehalts nach Injektion von Jodipinemulsion, dass soweit es sich um feine Fettemulsion handelt, es zum grössten Teil von der Leber festgehalten, während 
von Lunge und Milz nur wenig gebunden wird. Nomura ${ }^{5}$ sah auch die Leber als die Hauptablagerungsstätte des infundierten Fettes an, dann erst Skelettmuskulatur und subkutanes Fettgewebe.

Im Gegensatz zu dieser Auffassung konnte Mansfel $\mathrm{d}^{6)}$ in einem einschlägigen Versuch mit Olivenölemulsion zeigen, dass ein grosser Teil von intravenös injiziertem, fein zerstäubtem Fett lange in der Lunge vorgefunden wurde. DieserTatsache zufolge liess er der Lunge beim normalen Fettstoffwechsel eine wesentliche Rolle zukommen. Mit einem gleichen Verfahren hat auch Köszeg.7) die Fettablagerungsorgane der Reihe nach wie folgt bestimmt, zuerst Lunge, dann Leber und schliesslich Milz. Derman und Leites ${ }^{8 / 2}$ konnten bei Hunden nach enteraler und parenteraler Einfuhr von mit Neutralfett und Lipoid gemischter Oleinsäure die Fettsäure in Lunge, Leber und Milz konstatieren. Dagegen bemerkten sie bei enteraler Belastung mit Olivenöl eine Speicherung des Neutralfettes hauptsächlich in Alveolarepithelien der Lunge, Leber- und K u pffer schen Sternzellen und Retikuloendothelien der Milz. Sie meinten daraus, dass Lunge, Leber und Milz im Fett- und Lipoidstoffwechsel eine aktive Rolle spielen, indem eingeführte Fette bzw. Lipoide von ihnen gespalten werden, wobei möglicherweise aus deren Spaltprodukten andere Fette und Lipoide entstehen können. Dieser Auffassung schloss sich auch Kawamura durch ähnliche Experimente an. U. Kon ${ }^{10)}$ hat auch bei den intravenös mit Lanolinemulsion behandelten Kaninchen Ablagerung des Fettes in Leber, Milz, Knochenmark, Lunge und Nebenniere nachgewiesen. Bond i und $\mathrm{Ne}$ u man $\mathrm{n}$ beschuldigten Knochenmark und Leber als fettspeichernde Organe, indem sie eine deutliche Rotfärbung dieser Organe nach Injektion einer Suspension von mit Scharlachrot gefärbtem Fett beobachteten.

Alles in allem genommen besteht noch keine übereinstimmende Meinung über das Schicksal intravenös infundierten Fettes, was wahrscheinlich auf den verschiedenen Untersuchungsmethoden und besonders Dispersitätsgraden der injizierten Fettemulsion beruht. Histologische Untersuchung gab dabei der chemischen den Vorzug, weil man mit ersterer den Wanderungsprozess des injizierten Fettes im Tierkörper in Einzelheiten verfolgen kann, besonders wenn vorher gefürbtes Fett dafür zur Verfügung steht. Von diesem Gesichtspunkt aus habe ich in der vorliegenden Arbeit nach dem in dieser Klinik geübten Verfahren eine Emulsion von sehr hoher Dispersität mit gefärbtem Fett hergestellt. Mit dieser Fettemulsion habe ich mich bemüht, das in Frage kommende Problem von neuem zu ergründen. 


\section{Versuchsmethodik.}

Zum Versuch wurden bloss die Kaninchen gebraucht, die einige Tage vor dem Versuch mit bestimmter Nahrung (Tôfukara) gefüttert worden waren. Zur Herstellung der intravenös brauchbaren ölemulsion wurde das Olivenöl zunächst mit Scharlachrot gesättigt gefärbt, indem es mit dem Farbstoff vermischt, lange im Zimmer stehengelassen und danach der überschüssige Farbstoffteil mittels der Zentrifuge entfernt wurde. Das gefärbte Öl wurde dann mit einer kleinen Menge in Natrium carbonicum gelöster Protalbinsäure im Mörser zerrieben, tropfenweise mit destilliertem Wasser verdünnt, bis es eine ca. 17\%ige Aufschwemmung ausmachte und schliesslich durch den Homogenisator getrieben. In einigen Untersuchungen benutzte ich als Schutzstoff zur Emulgierung des Fettes Lezithin statt Protalbinsäure. Die so hergestellte Ölemulsion wurde den Kaninchen in die Ohrvene injiziert und in bestimmten Zeitabständen danach starben die Tiere durch Karotisschnitt den Verblutungstod. Die gleich danach aus Leber, Milz und Lunge herausgeschnittenen Stücke wurden in 5\% iger Formalinlösung fixiert, gefriergeschnitten und mit Hämatoxylin und Sudan III gefärbt und mikroskopisch untersucht.

\section{Versuchsergebnisse.}

Zur Untersuchung wurden insgesamt 16 Kaninchen gebraucht, die in 7 Gruppen geteilt wurden. Die Versuchstiere bekamen pro $\mathrm{kg} 10 \mathrm{ccm}$ der gefärbten Ólemulsion in die Ohrvene und wurden gruppenweise 30 Minuten, 1, 2, 3, 6, 12 und 24 Stunden nach der Injektion getötet.

A) 1. Gruppe (Kaninchen Nr. 1, 令, $2100 \mathrm{~g}, \mathrm{Nr} .2$, 令, $2070 \mathrm{~g}$, Nr. 3, 今, 1900 g), 30 Minuten nach der Ölinjektion getötet. Lunge : Zahlreiche rot gefärbte Fettröpfchen fanden sich frei in den Lungenkapillaren, nur vereinzelt aber die fettphagozytierten Endothelzellen. Leber: Auffallenderweise waren die rot gefärbten Fettröpfchen gar nicht in den eigentlichen Leberzellen, sondern ausschliesslich in den Kupfferschen Zellen nachweisbar, und zumal besonders reichlich in der Peripherie, nur spärlich dagegen im Mittelfeld der Leberlappen und in der Nähe der Zentralvenen. Milz: In den Sinusendothelzellen war eine grosse Zahl der rot gefärbten Fettröpfchen aufgenommen, nicht aber in den Lymphfollikeln. Die Phagozyten kamen in der Regel nicht vereinzelt, sondern gruppenweise vor.

B) 2. Gruppe (Kaninchen Nr. 4, 冬, $1800 \mathrm{~g}, \mathrm{Nr} .5, \hat{\delta}, 2280 \mathrm{~g}$, $\mathrm{Nr} .6$, $\delta, 1960 \mathrm{~g}), 1$ Stunde nach der Injektion getötet. Lunge: Ergebnis fast gleich wie bei der ersten Gruppe. Leber: Die mit rotge- 
färbten Fettröpfchen beladenen Zellen schienen etwas verminderter als bei der ersten Gruppe. Sie fanden sich zumeist in der Peripherie der Leberlappen, nur vereinzelt in der Zentralzone. In den Leberparenchymzellen fand man hier auch keine eingewanderten Fetttröpfchen wie zuvor. Milz: Die Phagozytose aufweisenden Sinusendothelzellen wurden stärker aufgequollen als bei der ersten Gruppe, sie enthielten gewöhnlich 2 oder 3 rot gefärbte Fettröpfchen, so dass die Kerne an den Rändern der Zellen gedrängt standen. Beim Tier Nr. 4 fanden sich zumal im Venensinus nicht selten sehr grosse Histiozyten, innerhalb welcher einige, rot gefärbte Fettröpfchen nachweisbar waren.

C) 3. Gruppe (Kaninchen Nr. 7, 令, $1900 \mathrm{~g}, \mathrm{Nr} .8$, 令, $1620 \mathrm{~g}$ ), 2 Stunden nach der Injektion getötet. Lunge: Man fand nur vereinzelt Alveolarepithelien, die mit Fettröpfchen beladen waren. Leber : Im allgemeinen war das Ergebnis wie bei der zweiten Gruppe; es waren also viele rot gefärbte Fettröpfchen in den Kupfferschen Sternzellen in der peripheren Zone der Leberlappen zu beobachten. Im Falle Nr. 7 erweiterten sich die Kapillaren hie und da, die mit den rot gefärbten Fettröpfchen beladenen Leukozyten prall gefüllt waren. Milz: Es sah fast gleich wie bei der zweiten Gruppe aus. Man bemerkt aber schon erst um diese Zeit freiliegende Pigmentkörner neben den Zelleinschlüssen.

D) 4. Gruppe (Kaninchen Nr. 9, 命, $1900 \mathrm{~g}, \mathrm{Nr} .10$, $\hat{o}, 1800 \mathrm{~g}$ ), 3 Stunden nach der Injektion getötet. Lunge: Es traten in der Lunge die Exsudatzellen auf, die feine rot gefärbte Fettröpfchen enthielten. Die in die Kapillaren embolisch gesteckten Fettröpfchen schienen sich jetzt zugunsten der in den Alveolenzellen eingeschlossenen allmählich immer mehr zu vermindern. Leber: Die gefärbten Fetttröpfchen, die in den $\mathrm{K}$ upfferschen Sternzellen aufgenommen wordenwaren, verminderten sich nun beträchtlich an Zahl, ohne dabei Pigmentschollen zum Vorschein zu bringen, was doch in der Milz der Fall war. Milz: Die gefärbten Fettröpfchen verliessen schon erheblich die Sinusendothelzellen, im Gegensatz dazu kam freie Farbstoffmasse hie und da angehäuft vor.

E) 5. Gruppe (Kaninchen Nr. 10, $\hat{o}, 1760 \mathrm{~g}$ und $\mathrm{Nr}$. 11, $\hat{o}$, $1880 \mathrm{~g}), 6$ Stunden nach der Injektion getötet. Lunge: In den Exsudatzellen beobachtete man um diese Zeit nur ausnahmsweise rot gefärbte Fettröpfchen. Letztere waren dagegen in den Alveolenzellen häufiger getroffen. Leber: In den Sternzellen waren mehr oler minter reichliche gefärbte Fettröpfchen noch immer nachweis- 
bar, dagegen wurden freie Pigmentschollen stets vermisst. Milz: Die Sinusendothelzellen hatten nur spärliche Fettröpfchen, die aber intensiver rot gefärbt wurden als zuvor. Dies beruht wahrscheinlich darauf, dass verhältnismässig mehr Farbstoff für Fettverfärbung in Betracht kommt, weil um diese Zeit das eingangs angehäufte Fett schon grösstenteils die Milz verliess, der Farbstoff aber beim Transport dem Fett nachschleppte und länger im Organe verweilte.

F) 6. Gruppe (Kaninchen Nr. 13, 合, $1500 \mathrm{~g}$ und Nr. 14, 今, $1620 \mathrm{~g}), 12$ Stunden nach der Injektion getötet.

Die gefärbten Fettröpfchen wurden in der Lunge recht vermindert. Auch in den Sternzellen waren nur wenige zu konstatieren. Aus der Milz ging die Pigmentausscheidung auch wie bei den vorigen Gruppen immer lebhafter vor.

G) 7. Gruppe (Kaninchen Nr. 15, 8, $1700 \mathrm{~g}$ und Nr. 16, b, $1720 \mathrm{~g}), 24$ Stunden nach der Infusion getötet.

In der Lunge bildeten sich stellenweise kleine Infarkte. Leber: In den Sternzellen waren gefärbte Fettröpfchen nicht mehr erkennbar, doch vereinzelt freie Pigmentschollen. In den Leberzellen war überhaupt keine gefärbte Masse wie vorher nachweisbar. Milz: In den Sinusendothelzellen beobachtete man keine gefärbten Fettröpfchen mehr, aber spärliche Menge von Pigmentschollen.

Das Fettgewebe, ebenso subkutanes wie mesenteriales, begann sich erst 12 Stunden nach der Ölinfusion an zu verfärben und tingierte sich allmählich immer stärker gelblichrot, bis es schliesslich nach 24 Stunden schön rot aussah. Auch durch mikroskopische Untersuchung wurde diese Beobachtung bestätigt, indem das frisch zerzupfte Fettge webe Anhäufungen der deutlich gefärbten Fettzellen aufwies, die man zumal nur in den späteren Versuchsstunden nachweisen konnte. Andererseits wurde eine bestimmte Menge Fettgewebe aus den Tieren von zwei letzteren Versuchsgruppen entnommen und mit einer Menge Alkohol behandelt. Der Extrakt verfärbte sich dabei je nach der Länge der Versuchsfrist immer stärker gelblichrot. Dies liess sich dabei nur schätzungsweise heobachten, es gelang aber nicht, die in die Extrakte übergegangene Menge des Farbstoffes kolorimetrisch genau zu bestimmen.

\section{Besprechung der Ergebnisse.}

Aus den Ergebnissen der oben angeführten Untersuchungen lässt 
sich etwas Wesentliches wieder im folgenden betreffs einzelner Organe zusammenstellen. Bei Verfolgung des Schicksals des intravenös eingeführten Fettes im Organismus hat die histologische Untersuchung im Vergleich mit der chemischen soviel Vorteil als bei letzterer in den Geweben physiologisch vorkommendes Fett von infundiertem und abgelagertem kaum zu unterscheiden ist, was aber durch mikroskopischen Nachweis der gefärbten Fettröpfchen leicht geschieht. Seitens der histologischen Untersuchung bleibt es allerdings auch ohne Nachteil, weil das infundierte, rot gefärbte Fett im Körper des Organismus durch biologische Wirkungen früher oder später verändert wird, indem der Farbstoff die Fettmasse verlässt, welche letztere so dann unserem Nachweise vollständig entgeht. Durch diese Untersuchung'smethode kann man deswegen das Schicksal infundierten Fettes nur soweit verfolgen, als die gefärbte Fettmasse in den Geweben noch ihre Tinktion behält. Ob dabei die Entfärbung: des Fettes gleichzeitig auch seine molekulare Veränderung, wie etwa enzymatische Zerlegung dokumentiert, das ist natülich nicht leicht zu entscheiden.

\section{Lunge.}

Kurz nach Injektion der Ölemulsion beobachtet man eine Zeitlang reichliche Fettröpfchen in den Kapillaren der Lunge, die mit der Zeit in die Endothelzellen der Kapillarwände übergehen. Ferner platzen die Gefässe und die herausgewanderten Fettröpfchen werden nachträglich von den Alveolarepithelien aufgenommen. Späterhin treten noch die Exsudatzellen in den Alveolen auf, die mit reichlichen feinen Fettröpfchen beladen sind. Auf diese Weise verschwinden die anfangs reichlich vorhandenen Fettröpfchen allmählich immer mehr aus der Lunge, bis schliesslich nur noch kleine Infark tbildungen hie und da bestehen. Injizierte Fettröpfchen strömen also nicht ganz glatt durch die Lungenkapillaren hindurch, sondern werden teilweise in diesen zurückgehalten und weiter veraibeitet.

Die Stagnation der Fettröpfchen in den Lungengefässen nach der intravenösen Darreichung wurde von vielen Autoren beobachtet. Derman und Leites fanden bei den mit Oleinsäure intravenös behandelten Tieren gelblich-orange farbene Massen stellen weise in Gefässen des Kapillartypus. Bei intravenöser Belastung der Kaninchen mit Cholesterin-Olivenölgemisch bestätigte $\mathrm{Kaw}$ a mura reichliche isotrope Fettröpfchen und wenige Cholesterintafeln in den Lungenkapillaren. Auch beobachteten Kon und Yamada $a^{11}$ in ihrem Ver- 
such mit der Lanolinemulsion reichliche Tropfen in den Lungengefässen, welche damit bald embolisch verstopft, bald aber nur vorübergehend gefüllt werden. Reuter ${ }^{12}$ vertritt die Ansicht, dass die zirkulierenden Fette leicht in die Lungengefässe zusammenströmen, weil das Lungengewebe so grob gebaut und infolgedessen sehr dehnbar, ausserdem der Blutdruck in den Lungenkapillaren viel niedriger als der des grossen Krreislaufs ist.

Der Befund der Fettstagnation in den Lungengefässen verleitet manchmal die früheren Autoren anzunehmen, dass die Lunge aktiv am Fettstoffwechsel beteiligt ist. Diese Meinung wurde nicht nur seitens der Anatome sondern manchmal auch von den Chemikern erhoben. So erörterten Sieber, ${ }^{13)}$ Pagenstecher ${ }^{14)}$ u. a., dass der Lungenextrakt die Fähigkeit besitzt, Neutralfette zu zerlegen. Die Beweiskraft der von diesen Autoren gemachten Versuche wurde aber später von Freudenberg ${ }^{157}$ und $A \mathrm{maki}^{16)}$ widerlegt. Dieser Autor, der seine Arbeit in unserer Klinik ausführte, wies besonders auf die Fehlerquelle der Kanitzschen Untersuchungsmethode hin, die die genannten Autoren verwendet hatten, bei welcher die in den Verdauungsprodukten beigemischten Aminosäuren durch direkte Titrierung mit Alkali im alkoholischen Medium ebenso gut Säurewerte wie gespaltene Neutralfette liefern können.

Die anderen Autoren, die auch durch chemische Untersuchung an die Teilnahme der Lunge am Fettstoffwechsel glauben, basieren ihre Annahme auf die Tatsache, dass sie eine grosse Menge von intravenös einverleibtem Fett in der Lunge nach weisen konnten (Mansfeld, Köszeg). Man muss aber dabei natürlich an ein Zustandekommen der embolischen Stagnation des eingeführten Fettes denken, die man bei der chemischen Untersuchung garnicht von der aktiven Zurückhaltung des Fettes seitens der Lunge unterscheiden könnte. In dieser Hinsicht spricht aber Köszeg aus, dass die Lunge imstande ist, die infundierten Fette eine Weile in sich zurückzuhalten, um zur Verarbeitung der resorbierten Fette beizutragen, auch wenn eine Aufschwemmung von so fein zerteilten Fettröpfchen, die kleiner als Erythrozyten sind und die Lungenkapillaren glatt durchpassieren mögen, verwendet wird. G.S S t to ${ }^{17)}$ nahm in dieser Klinik eine Untersuchung über die Emboliebildung nach Fettinfusion vor, indem er selbst Emulsionen von verschiedenen Dispersionen herstellte. Bei Behandlung der Tiere mit einer Emulsion von gröberen Partikelchen fand er natürlich einen grösseren Fettgehalt der Lunge als beim Versuche mit einer höher dispergierten Emulsion. Allerdings selbst beim 
Gebrauch einer äusserst fein dispergierten Emulsion kam doch eine kleine Vermehrung des Fettgehaltes seitens der Lunge zustande, die natürlich immer als Folge der Emboliebildung betrachtet werden muss. Gewiss hat Klot $z^{18}$ Recht, wenn er beim Versuch mit Olivenölcholesterinemulsionen sagte, dass feine Fettpartikelchen ohne Hindernisse durch die Lunge passieren, aber grössere, welche sich in den Kapillaren embolisch finden, durch die Serum-oder Zellipase weiter verdaut werden.

Die embolische Verstopfung der Lungenkapillaren durch die infundierten Fettröpfchen konnte ich auch auf meinen Präparaten gut bestätigen. Die Infarktbildung, die in den späteren Versuchsstunden erschien, spricht zweifellos für die bestandene Embolie. Der Emboliebildung folgt natürlich eine Fettstagnation stromaufwärts der verschlossenen Stelle. Dies ruft die herdförmige Lokalisation der kleinen Fettanhäufungen hervor, wie ich eben in meinen Präparaten beobachtete. Eine gleichmässige Verteilung der Fettröpfchen über das ganze Gewebe, wie man beim aktiven Beteiligen der Organe als Abfangsapparat vielleicht vermuten könnte, kam dabei niemals zustande.

$\mathrm{Zu}$ dieser Untersuchung habe ich auch eine hoch dispergierte Fettaufschwemmung gebraucht, die unter 20 Atmosphärendruck homogenisiert worden war. Die einzelnen Fettröpfchen waren mikroskopisch untersucht, sicher kleiner als Erythrozyten, wie Köszeg bei einer von ihm angewendeten Fettemulsion ausgesprochen hat. Jeder Forscher, der sich mit einer sehr feinen Fettemulsion beschäftigt zu haben glaubt, muss wohl einräumen, dass grössere Tropfen als Erythrozyten, wenn auch an $Z$ ahl recht klein, doch unvermeidlich darin. beigemengt sein können. Es ist also leicht verständlich bei intravenöser Infusion solcher Emulsion, dass die gröberen Fettröpfchen in den Lungenkapillaren stecken bleiben und dann stromaufwärts eine Menge Fettröpfchen, wenn sie auch genug fein sind und den Lungenfilter glatt durchpassieren können, hinter diesen haltmacht. Aus den so entstandenen Embolien können die gefesselten Fettröpfchen, wie es G. Sato behauptet, zum Teil wieder gelöst und in den Kreislauf vorgeschleudert werden. Die anderen werden aber zweifellos lange festgehalten und inzwischen von den Histiozyten verarbeitet. Wenn man dabei bloss aus der Erscheinung der Fettphagozytose seitens der Lungenhistiozyten diesem Organ die Bedeutung als ein Abfangsorgan wie andere retikuloendotheliale Gewebe zumessen will, so muss man vorläufig den mechanischen Vorgang der Emboliebildung voll- 
ständig ignorieren. Natürlich kann aber die aktive Fettphagozytose, wenn überhaupt, unabhängig von der Embolie von statten gehen. Wie viel dann von stagniertem Fett dabei primär oder sekundär zurückgehalten wird, das lässt sich natürlich nicht ermessen.

\section{Leber.}

Manche Autoren behaupten, dass die Leber beim Fettstoff wechsel eine wichtige Rolle spielt. Die chemischen Untersuchungen von Le athes, ${ }^{19)}$ Joannovics und Pick, ${ }^{20)}$ Brown und Morris ${ }^{91)}$ u. a. haben gezeigt, dass die Leber die Zentralstätte für den Umbau der Neutralfette in die leicht oxydablen, weniger gesättigten Formen darstellt. Anitschkow ${ }^{22)} \mathrm{Zinserling},{ }^{237}$ Schilling, ${ }^{24}$ U. Kon, Kawamura, J. $\mathrm{Kon}^{25)}$ u. a., die diese Frage histologisch untersuchten, stimmen darin überein, dass die Sternzellen der Leber besonder's dafür verantwortlich sind. Bei meiner Untersuchung wurden auch die gefürbten Fettröpfchen schon früh nach der Infusion reichlich in den Sternzellen gefunden und verliessen erst in den späteren Stunden diese Stelle, wobei nur eine geringe Menge Farbstoffschollen zurückgelassen war. Auffallenderweise gelangen aber die gefärbten Fettröpfchen während der ganzen Versuchsdauer niemals in die eigentlichen Leberzellen, wenn auch solches Bild nicht selten an den dick geschnittenen Präparaten vorgetäuscht werden kann, beim Beobachten der schief getroffenen Zelleibreste der Sternzellen, die keinen Kern, wohl aber viele bunte Körnchen aufweisen. Die eigentümlich längliche Figur der Sternzellen lässt sich dabei durch geeignete Einstellung des mikroskopischen Gesichtsfeldes leicht beobachten, wodurch man gewöhnlich dieZugehörigkeit der Fettröpfchen richtig beurteilen kann. Derman und Leites beschrieben allerdings, dass sie reichliche Oleinsäurekörper im Protoplasma der Leberzellen der intravenös behandelten Tiere fanden. Diese Beobachtung ist aber mit den Ergebnissen vieler anderer Forscher nicht vereinbar. Bekanntlich sind auch die Leberzellen der unvorbehandelten Laboratoriumtiere in der Regel mit mehr oder minder reichlichen, durch Sudan leicht färbbaren Fettröpfchen versehen (Nakase u. Kikuchi $\left.{ }^{9^{(6)}}\right)$. Derartige Fettröpfchen lassen sich z. B. durch langfristige Karenz der Tiere gar nicht beseitigen. Bei dieser Sachlage ist man natürlich nicht imstande, das durch künstliche Einverleibung in den Geweben abgelagerte Fett von präformiertem zu unterscheiden, wenn die beiden Arten Fett mit Fettfarbstoffen gleich dargestellt werden. In der vorliegenden Arbeit 
waren die infundierten Fettröpfchen vorher rot gefärbt, so dass sie schon an den ungefärbten Präparaten erkannt werden und wenn nötig beim Gebrauch anderer Farbstoffe, z. B. von Nilblau unschwer von präformierten Tropfen getrennt werden können. Somit beweist das Ergebnis meines Versuches eindeutig, dass die intravenös infundierten, gefärbten Fettröpfchen, sowit es die Leber anbelangt, direkt nur in die Sternzellen, nicht aber in die Leberzellen gelangen. Wie sich die einınal in den Sternzellen aufgenommenen Fettröpfchen weiter verhalten, ob sie nicht sekundär in die Leberzellen übergehen, darauf gibt das Ergebnis meiner Untersuchung keine Antwort. Allerdings kann man soweit mit Wahrscheinlichkeit sagen, dass sie gewisse Umwandlung in den Sternzellen erleiden, ehe sie diese Zelle verlassen, weil sie dabei, wie es in der Milz der Fall ist, nach langem Verweilen Pigmentreste zurücklassen.

\section{Milz.}

In meiner Untersuchung wurden reichliche Fettröpfchen in den Sinusendothelzellen der Milz gebunden, dagegen in den Lymphfollikeln nur wenig. Dadurch wurde der von U. Kon und Yamada, Kawamura und Derman und Leites bei der Lipoidinjektion erhobene Befund bestätigt, nur abgesehen davon, dass in meinem Versuch die genannten Milzzellen stets nit einer grösseren Menge Fetttröpfchen beladen waren als die Sternzellen, während dieses Verhältnis bei den Versuchen der anderen Autoren umgekehrt der Fall war. Mit meinem Versuchsergebnis deckt sich auch die Arbeit Otak as in dieser Klinik, der bei der intraperitonealen Einführung der rot gefärbten Ölemulsion mehr Ablagerung in den Zellen der Milzpulpa, als in den Sternzellen beobachtete. Entsprechend dem abgelagerten Fett erweist sich auch die Menge der abgestossenen Pigmentschollen, die in der Milz stets weitaus grösser als in der Leber gefunden war.

Der Befund der zurückgelassenen Farbstoffreste wurde aber von U. Kon und Yamada, und auch von Bondi und Neumann nicht besonders beachtet, die doch ebenfalls mit gefärbtem Fett arbeiteten.

Fettgewebe.

Das Mesenterial- sowie Subkutanfettgewebe wurde erst in den spüteren Stunden nach der. Injektion rot gefärbten Fettes verfärbt gefunden. Die Tinktion wurde mit der Zeit allmählich immer inten- 
siver, bis 24 Stunden nach der Fettinfusion, wo die in Leber und Milz abgelagerten Fettröpfchen schon grösstenteils diese Organe verliessen. U. Kon und Yamada bemerkten auch bei wiederholter Injektion sudangefärbter Ölemulsion allmähliche Rotfärbung des Subkutan- und Peritonealfettgewebes. Im Gegensatz zur Eiweissassimilation, bei welcher Nahrungseiweiss erst nach vollkommener Zerlegung in die einfachen Bausteine wieder in körpereignes Eiweiss umgebildet wird, verhält sich der Fettstoff wechsel sehr einfach, indem oral eingeführtes Fett schon als solches in den Depots abgelagert werden kann, ohne dabei seine Beschaffenheit allzu sehr umzuwandeln, wie es schon aus den Experimenten von Lebedeff, ${ }^{(8)} \mathrm{Munk},{ }^{929}$ Rosenfeld, ${ }^{30)} \mathrm{Jacobstal}^{31)}$ u. a. bekannt ist. Dieser Weg der Fettassimilation gilt auch für die parenterale Fettzufuhr; so fand No m ura in dieser Klinik, dass die Depotsfette der Kaninchen, die lange mit Lebertranemulsion intravenös behandelt wurden, schliesslich eine dem Lebertran sehr nahe Zusammensetzung übernahmen. In diesen Experimenten ist aber der Wanderungsprozess des einverleibten Fettes nicht näher erklärt.

Durch die vorliegende Untelsuchung wurde aber sichergestellt, dass intravenös dargereichtes Fett nicht sofort die Fettdepots erreicht, sondern ein gewisser Zeitraum stets dazu erforderlich ist. Welche Bedeutung man dieser Latenzzeit zumessen soll, ist vorläufig schwer zu entscheiden. Der zeitliche Verlauf der Versuchsserien spricht aber immer für die Annahme, dass einverleibtes Fett zuerst in den retikuloen dothelialen Organen eine Zeitlang verweilt und dann weiter in die Fettdepots übergeht. Das Fett unterliegt vielleicht in den Zellen der genannten $Z$ wischenstationen einer gewissen Veränderung, wie der zurückgelassene Farbstoff vermuten lässt. Die Entfärbung des Fettes im retikuloendothelialen System ist aber dabei keineswegs vollständig. Die nachträgliche Einwanderung des gefärbten Fettes in die Fettdepots weist immer auf dieses Verhältnis hin und auch aus dieser Beobachtung lässt sich wohl annehmen, dass nach intravenöser Fettinfusion unverändertes oder verhältnismässig weniger umgewandeltes Fett schliesslich die Fettdepots erreicht.

\section{Zusammenfassung.}

Aus der histologischen Untersuchung ergibt sich folgendes:

1. Bei intravenöser Einfuhr einer Ölemulsion von hoher Dispersität werden die Fettröpfchen nur wenig von der Lunge, dagegen 
sehr reichlich von der Milzpulpa und den Kupfferschen Sternzellen zurückgehalten.

2. In der Lunge verursachen gewöhnlich die gröberen Fettröpfchen Embolien, die nicht selten zur Infarktbildung führen. Wenn die Kapillaren lange verschlossen sind, werden dann Kapillarendothelund Exsudatzellen phagozytär wirksam. Eine aktive Beteiligung der Lunge am Fettstoffwechsel ist keineswegs erwiesen.

3. Die in den retikuloendothelialen Zellen abgefangenen Fettröpfchen entgehen im Laufe von einigen Stunden vollständig dem Nachweise, wobei Schollen des Farbstoffs, womit das infundierte Fett gefürbt war, zurückgelassen sind. Der Übertritt der injizierten Fetttröpfchen in die eigentlichen Leberzellen ist niemals nachweisbar.

4. In die Depotfettgervebe wandert das injizierte Fett erst nachträglich ein, dies geschieht zumal erst auf dem Umweg der retikuloendothelialen Gewebe.

\section{Literatur.}

(1) Bondi u. Neumann, Wien. kl. W., 1910, 734 .

(2) Saxl u. Donath, Wien. kl. W., 1924, 635.

(3) Nomura, Tohoku Journ. Exp. Med., 1929, 12, 247.

(4) Bodo u. Schäffer, Archiv f. exp. Path. u. Pharmak., 1927, 124, 326.

(5) Nomura, Tohoku Journ. Exp. Med., 1929, 12, 497.

(6) Mansfeld, Wien. kl. W, 1918, 776.

(7) Köszeg, Archiv f. exp. Path. u. Pharmak., 1927, 101, 305.

(8) Derman u. Leites, Virchows Archiv, 1928, 268, 440.

(9) Kawamura, Morphologie und Physiologie der Cholesterinsteatose, Jena 1927 .

(10) Ko n, Nisshin Igaku, 1916, 4, 1261.

(11) K o n u. Y a mada, Nihon Byorigakkai Kaishi, 1926, 6, 411.

(12) Reuter, Zitiert nach Kon und Y a mada.

(13) Sieber, Zt. f. physiol. Chem., 1908, 55, 177.

(14) Pagenstecher, Bjochem. Zt., 1908, 18, 285.

(15) Freudenberg, Biochem. Zt., 1912, 45, 467.

(16) A maki, Tohoku Journ. Exp. Med., 1924, 5, 13

(17) Sato, Tohoku Journ. Exp. Med., 1931, 18, 20.

(18) Klotz, Journ. of Medical Research, 1915-16, 33, 157.

(19) Leathes, Journ. of Physiol, 1909, 38, 38.

(20) Joannovics u. Pick, Wien. kl. W., $1910,573$.

(21) Brown u. Morris, Journ. Nutrit., 1930, 2, 509.

(22) Anitschkow, Beitr. z. pathol. Anat. u. z. allg. Pathol., 1913, 57, 201, Med Ki., 1914,465 .

(23) Zinserling, Beitr. z. pathol. Anat. u. z. allg. Pathol., 1923, 71, 292.

(24) Schilling, Virchows Archiv, 1909, 196, 1.

(25) Ko n, Chosen Igakkai Zasshi, 1932, 22, 831 u. 1933, 23, 265. 
(26) Nakase n. Kikuchi, Chugai Iji Shimpo, 1925, Nr. 1080, 499.

(27) Otak a, Toboku Journ. Exp. Med., 1935, 26, 482.

(28) Le bedeff, Archiv f. d. ges. Physiol., 1883, 31, 11.

(29) Munk, Archiv f. Physiol., 1883, 31, 273.

(30) Rosenfeld, Verh. d. Kongress. f. inn. Med., 1901, 19,518, Verh. d. Dtsch. Pathol. Ges., 1903, 6, 73.

(31) Jacobstal, Verh. d. Dtsch. Pathol. Ges., 1909, 13, 380. 